\title{
Reducción de los síntomas de ansiedad y sensibilidad a la ansiedad mediante la aplicación de un programa de meditación mindfulness
}

\author{
Laura YAGÜE, Ana Isabel SÁNCHEZ-RODRÍGUEZ, Israel MAÑAS, Inmaculada \\ GÓMEZ-BECERRA y Clemente FRANCO
}

Universidad de Almería, España

(Received on September 28, 2014; Accepted on July 30, 2015)

RESUMEN: El objetivo del presente estudio es examinar la eficacia de un programa de entrenamiento en mindfulness para reducir los niveles de ansiedad. La muestra estuvo constituida por 20 participantes aquejados de ansiedad con puntuaciones elevadas en ASI-3 y en la subescala de ansiedad del SCL-90. Se realizó un diseño cuasiexperimental de comparación de grupos con medición pretest-postest, con un grupo experimental y un grupo control con 10 participantes cada grupo. Como instrumentos de evaluación se utilizaron los cuestionarios ASI-3, MAAS y SCL-90. Los análisis estadísticos muestran una reducción en todas las dimensiones del factor Sensibilidad a la ansiedad y también en la subescala de ansiedad del Scl-90 una vez finalizada la intervención, así como una mejora en la capacidad de estar presentes o atención plena medida a través de la escala MAAS. Estos resultados son acordes con otras investigaciones donde se ha comprobado la eficacia de las técnicas de mindfulnessen la mejora de los síntomas de ansiedad.

Palabras clave: meditación, mindfulness, conciencia plena, ansiedad, sensibilidad a la ansiedad, intervención.

\section{Improvement of the symptoms of anxiety and anxiety sensitivity through the application of a mindfulness meditation}

\begin{abstract}
The aim of this study is to examine the effectiveness of a training program mindfulness to reduce anxiety levels. The sample consisted of 20 participants suffering from anxiety and high scores on ASI-3 and subscale of anxiety from SCL-90. A quasi-experimental comparison group design with pretest-posttest measurement with an experimental group and a control group with 10 participants each group was performed. As instruments of assessment questionnaires ASI-3, MAAS, and SCL-90 were used. Statistical analyzes show a reduction in all in dimensions of anxiety sensitivity factor and anxiety subscale of the SCL-90 once the interventions as well as improvement in the ability to be present or mindfulness measured by the MAAS scale. The results are consistent with other research which has show the effectiveness of mindfulness techniques in improving the symptoms of anxiety.
\end{abstract}

Keywords: Meditation; mindfulness; full awareness; anxiety; anxiety sensitivity.

Correspondencia: Inmaculada Gómez. Universidad de Almería. Cañada de San Urbano. Almería, 04120. Email: igomez@ual.es. 


\section{Introducción}

El término ansiedad alude a la combinación de distintas manifestaciones físicas y mentales que no son atribuibles a peligros reales, manifestados en forma de crisis o bien como un estado persistente y difuso, pudiendo llegar al pánico, se relaciona con la anticipación de peligros futuros, indefinibles e imprevisibles (Marks, 1986). Se considera un mecanismo evolucionado de adaptación que potencia la supervivencia de nuestra especie (Thyer, 1987). La característica más llamativa de la ansiedad es su carácter anticipatorio, es decir, posee la capacidad de prever o señalar el peligro o amenaza para el propio individuo, confiriéndole un valor funcional importante (Sandíny Chorot, 1995). Sin embargo, cuando la ansiedad supera los índices de normalidad en los parámetros de intensidad, frecuencia o duración, o bien, se relaciona con estímulos no amenazantes para el organismo, provoca manifestaciones patológicas en el individuo, tanto a nivel emocional como funcional (Vila, 1984). De hecho, en la actualidad, los síntomas y trastornos de ansiedad son los problemas psicológicos más prevalentes, tanto en adultos como en niños y adolescentes (Sandín, Valiente yChorot, 2009). La Sensibilidad a la Ansiedad (SA), un constructo muy relacionado con la ansiedad, el cual hace referencia a la creencia de que experimentar sensaciones corporales asociadas con la ansiedad o el miedo, provocan consecuencias adversas o indeseables, como el desarrollo de enfermedades o el rechazo social (Reiss y McNally, 1985). Es la (SA), el miedo a experimentarsensaciones propias de la sintomatología del trastorno de ansiedad (Reiss, Peterson, Gursky y McNally, 1986) y se relaciona con los trastornos asociados a la ansiedad y el pánico (Sandín, 2005).

Mindfulness ha adquirido una gran importancia en su vertiente de aplicación terapéutica, comenzando a ser incluida y reconocida como un componente fundamental en las terapias actuales. Algunos autores (Hayes, 2004; Baer, 2006; Hayes, Luoma, Bond, Massuda y Lillis, 2006) han denominado a estas terapias basadas en la aceptación y enmindfulness como "terapias de tercera generación", sugiriendo la emergencia de un nuevo paradigma(véase Hayes, 2004a, 2004b; Mañas, 2007; Pérez-Álvarez, 2008; Vallejo, 2007). Así, Hayes (2004), considera la tercera generación de terapias de conducta como fundamentada en una aproximación empírica y enfocada a los principios de aprendizaje. Considera que la tercera generación de terapias cognitivas y conductuales es particularmente sensible al contexto y a las funciones de los fenómenos psicológicos y no solo a la forma de estos fenómenos psicológicos. Estos tratamientos buscan la construcción de repertorios de funcionamientos amplios, flexibles y efectivos para la persona, en lugar de procurar el intento por eliminar, ignorar o cambiar determinados eventos privados negativos. Entendiendo que este intento o conjunto de estrategias cognitivas de control, eliminación o cambio estarían paradójicamente a la base del desarrollo y mantenimiento de determinados trastornos psicológicos. Desde las terapias de tercera generación se aboga, por tanto por la aceptación, el autoconocimiento y la redirección de la personahacia sus parcelas vitales valiosas.

El término mindfulness no tiene una traducción exacta al español, y aunque se han propuesto términos como "atención y consciencia plena”, el término mindfulness es el más usado en el ámbito científico (ver Prieto, 2007).

Con el objetivo de realizar una definición operativa de mindfulness, Bishop et al. (2004) diferencian dos tipos de componentes implicados. El primer componente es el de la 
autorregulación de la atención. El objetivo principal de este componente es el mantenimiento y la redirección de la atención, implicando además la conducta de selección de estímulos concretos. De este modo, se incrementa el reconocimiento de los eventos mentales en el momento presente. Los procesos que incluye mindfulnesso la atención plena son: (a) atención sostenida; (b) conmutación de la atención; e, (c) inhibición de la elaboración de pensamientos, sentimientos, sensaciones y emociones. El segundo componente propuesto por estos autores es la orientación hacia la experiencia. Este componente implica adoptar un tipo de relación particular hacia las propias experiencias en el momento presente. Este tipo de relación se caracteriza por una actitud de curiosidad, apertura y aceptación. De este modo, se aprende a no reaccionar automáticamente a la estimulación que se esté percibiendo.

Según Kabat-Zinn (2003) la atención plena es la conciencia que surge de prestar atención, de forma intencional, a la experiencia tal y como es en el momento presente, sin juzgarla y sin reaccionar a ella. De este modo, la práctica de la meditación, va a permitir incrementar la flexibilidad cognitiva, así como la capacidad de responder de modo más reflexivo a las situaciones, en lugar de funcionar en "piloto automático". Los elementos fundamentales del mindfulness propuestos por Germer (2005) son: la consciencia sobre los eventos privados, la capacidad para situarse en el momento presente y la aceptación de esos eventos privados.

En el ámbito del mindfulness se diferencia, especialmente, el programa de entrenamiento psicoeducativo desarrollado por Jon Kabat-Zinn: Mindfulness-Based Stress Reduction (MBSR; Kabat-Zinn, 1982, 1990, 2003). MBSR es un programa de entrenamiento general, no específico para ningún trastorno psicológico, cuya aplicación ha demostrado ser efectiva en múltiples problemas tanto en el terreno de la salud mental como física, en pacientes, así como también en profesionales de la salud (e.g., véanse recientes y exhaustivas revisiones en Chiesa y Serreti, 2009; Irving, Dobkin y Park, 2009; véase también Grossman, Niemann, Schmidt y Walach, 2004).

Desde hace unos treinta años, mindfulness ha comenzado a entrenarse en contextos clínicos y existe abundante evidencia empírica que ha puesto de manifiesto sus beneficios en el tratamiento de condiciones relacionadas con el estrés, la depresión, la ansiedad y la salud en general (Kabat-Zinn, 2005).

Los hallazgos obtenidos en diversos estudios han demostrado que la práctica regular y continua de la meditación produce diferentes efectos beneficiosos sobre el organismo, algunos de ellos relacionados con la ansiedad (Solberg, Ingjer, Holen, Sundgot-Borgen, Nilsson y Holme, 2000), reducción de la tensión arterial (Barnes, Treiber y Davis, 2001), mejora significativa del insomnio (Jacobs, Benson y Friedman, 1993), reducciones de los niveles de nerviosismo, preocupación, malestar emocional y estrés e incrementos de los niveles de relajación muscular, tranquilidad emocional y del grado de bienestar general (Mañas, Luciano y Sánchez, 2008; Mañas, Sánchez y Luciano, 2008; Franco, Mañas, Cangas, Moreno y Gallego, 2010), entre otros.Por todo ello, se han desarrollado intervenciones basadas en mindfulness para tratar trastornos específicos como depresión, adicciones, trastornos de la conducta alimentaria, trastornos de ansiedad, trastornos relacionados con el dolor corporal y la salud en general (Orsillo, Roemer, Block, Lejeune y Herbert, 2004; Orsillo, Roemer, BlockLerner y Tull, 2004, Franco, Mañas y Justo 2010). Los datos preliminares de investigaciones 
con estas técnicas han aportado cierta evidencia en apoyo de esos tratamientos (Baer, 2003; Roemer y Orsillo, 2004). Tal es así, que el Instituto Nacional de Salud de EEUU recomienda el uso de la meditación como tratamiento eficaz en diversos trastornos psicopatológicos y médicos, entre ellos la ansiedad (Cerlin y Lee, 1997). Tras realizar un meta-análisis de la efectividad de mindfulnessen el tratamiento de la ansiedad y depresión el Ministerio de Salud (2007), asegura que las técnicas de mindfulnessresultan efectivas tanto por sí solas cómo incluidas dentro de un paquete terapéuticopero que aún queda seguir estudiando sus efectos y construir estudios más controlados y rigurosos que permitan ver el desarrollo y el mantenimiento de sus efectos positivos a lo largo del tiempo.

Estos tratamientos se han aplicado tanto individualmente como de manera grupal. Evans et al. (2008) han constatado en un grupo de personas, mejoras significativas en los niveles de ansiedad, preocupación y sintomatología depresiva, alcanzando niveles no clínicos. Por otro lado, Roemer y Orsillo (2003, 2007) obtuvieron resultados positivos con la aplicación de un programa basado en mindfulness en personas con trastorno de ansiedad generalizada. Sin embargo, ambos estudios carecen de grupo control.

Posteriormente, Roemer, Orsillo y SaltersPedneault, (2008) en un estudio controlado aleatorizado con 31 sujetos, encontraron mejoras en los niveles de ansiedad, preocupación, evitación de las emociones, calidad de vida y en mindfulness. Estos cambios se mantuvieron en el seguimiento a los 3 y 9 meses. Kim et al. (2009) han publicado un estudio controlado aleatorizado utilizando mindfulness con 46 pacientes con trastorno de ansiedad generalizada, que se comparan con un grupo control sometido a psicoeducación sobre la ansiedad. Entre los resultados de este estudio se señala una reducción significativa de los síntomas de ansiedad y depresión en el grupo mindfulness.

Recientemente ha sido aplicado un programa de entrenamiento en mindfulness a docentes, donde se obtuvo una reducción significativa tanto en los niveles de estrés docente, como en el número de días de baja laboral en el grupo experimental en comparación con el grupo control (Mañas, Franco y Justo, 2011). Los resultados obtenidos en este estudio indican la idoneidad de la práctica de mindfulness en el ámbito docente, donde son frecuentes los problemas de ansiedad, estrés y depresión. La disminución de los niveles de estrés y aumento del bienestar y afrontamiento del estrés repercutieron en una disminución de días de baja laboral.

En resumen, existe una creciente evidencia empírica que constata la eficacia de la aplicación las habilidades mindfulnessen el contexto de la ansiedad (Lykins y Baer, 2009; Williams, 2010). Y, aunque la efectividad de las intervenciones basadas en mindfulness en adultos son numerosas (Baer, 2003; Hayes, Follette y Linehan, 2004; Germer, Siegel y Fulton, 2005), en España las investigaciones sobre mindfulness se están iniciando recientemente. A través del presente estudio basado en mindfulness esperamos que se produzca una reducción en los síntomas de ansiedad y la sensibilidad hacia la misma entre los sujetos participantes. Nuestra hipótesis de investigación es que los síntomas de ansiedad de las personas que componen el grupo experimental, mejorarán significativamente mediante la práctica de mindfulness en comparación con el grupo control en las variables, síntomas de ansiedad, sensibilidad a la ansiedad y en atención consciente. 


\section{Método}

\section{Participantes}

En el presente estudio participaron un total de 20 personas aquejadas de ansiedad con puntuaciones elevadas en ASI-3 y en la subescala de ansiedad del SCL-90, con una edad de entre 20 y 40 años y de ambos sexos (13 mujeres y 7 hombres) $(M=29,85$ y $D T=10,75)$. De esas 20 personas participantes, 10 formaron parte del grupo experimental y 10 del grupo control. La asignación de los sujetos a uno u otro grupo se realizó al azar de manera aleatoria.El grupo control estaba formado por 6 mujeres y 4 hombres y el experimental por 7 mujeres y 3 hombres.

\section{Instrumentos}

Para la evaluación de la sensibilidad a la ansiedad, se empleó la escala ASI-3.Índice de Sensibilidad a la Ansiedad, (Peterson y Reiss, 1992). Se aplicó la versión española (Sandín, Valiente y Chorot, 1999), que al igual que la versión original, consta de 16 ítems y mantiene su misma estructura general y el formato de respuesta. El participante debe indicar el grado en que experimenta cada uno de los 16 enunciados según una escala tipo Likert, pudiendo variar entre «Nada en absoluto» (0) y «Muchísimo» (4). Los ítems hacen referencia a reacciones de miedo/ ansiedad ante la experiencia de síntomas de tipo somático (p.ej., «Me asusto cuando mi corazón late de forma rápida »), sociales («Para mí es importante no dar la impresión de estar nervioso»), y síntomas de descontrol cognitivo (p.ej., «Me asusto cuando soy incapaz de mantener mi mente concentrada en una tarea»). Esta prueba posee excelentes propiedades psicométricas, tanto con población clínica como no clínica (Sandínet al., 1996, 2001) y una adecuada consistencia factorial (Sandín et al., 2001, 2004). Además de la obtención de una puntuación general, derivamos tres subescalas: (1) ASI-somática, (2) ASIcognitiva y (3) ASIsocial.

La evaluación de la atención consciente se evaluó con la escala MAAS. Escala de Atención Consciente, (Brown y Ryan, 2003). Es un autoinforme que evalúa, con una única puntuación, la capacidad del individuo para estar atento y consciente de la experiencia del momento presente en la vida cotidiana. Se puede utilizar sin que el sujeto tenga experiencia previa en meditación y la versión original presenta unas buenas propiedades psicométricas. Es la herramienta más utilizada en estudios de investigación donde se pretende evaluar mindfulness. Consta de 15 ítems y se puntúan según una escala Likert con un rango entre 1 (casi siempre) y 6 (casi nunca). Ha sido validada al castellano obteniendo buenos resultados, alfa de Crombach de 0,89 y una varianza total de 42,8\%, encontrándose resultados similares a la escala original (Soler et al. 2012).

La evaluación de los síntomas de ansiedad se llevo a cabo utilizando la subscala de ansiedad del SCL-90-R. Inventario de Síntomas de Derogatis, Revisado por Derogatis y asociados $(1975,1994)$. Es un instrumento de autoinforme desarrollado para evaluar el grado de malestar psicológico actual que experimenta una persona. Consiste en un listado de 90 síntomas psiquiátricos de variado nivel de gravedad, frente a los cuales la persona debe indicar en qué medida lo han molestado o perturbado cada uno de esos problemas durante el período comprendido entre el día de la evaluación y la semana inmediatamente anterior. Para responder, se ofrece una escala de 0 a 4 puntos. Una vez puntuadas las respuestas es posible 
caracterizar la sintomatología del evaluado en un perfil compuesto por nueve dimensiones primarias de síntomas (Somatización, Obsesiones, Sensitividad Interpersonal, Depresión, Ansiedad, Hostilidad, Ansiedad Fóbica, Ideación Paranoide y Psicoticismo) y tres índices globales de psicopatología: el Î́ndice de Gravedad Global, el Índice de Malestar Positivo y el Total de Síntomas Positivos.La subescala de ansiedad evalúa la presencia de signos generales de ansiedad tales como nerviosismo, tensión, ataques de pánico, miedos.Sus propiedades psicométricas han resultado apropiadas y estables a través del tiempo, países, grupos de edad y tipos de población evaluada (pacientes versus no pacientes). La fiabilidad de las nueve dimensiones alcanza valores de $r=0.70$ y consistencia interna 0.80 (Derogatis y Savitz, 2000). Otros estudios han demostrado una satisfactoria escalabilidad de las dimensiones (Olsen. Mortensen y Bech, 2004; Schmitz et al., 2000) y muestran un comportamiento psicométrico idóneo cuando se analizan con modelos de Teoría de Respuesta al Ítem (Olsen et al., 2004).

\section{Diseño y variables}

Realizamos un ensayo clínico controlado con grupos paralelos, se establecieron dos grupos; el grupo experimental y el grupo control, la asignación de los participantes a uno u otro grupo se realizó de manera aleatoria. Utilizando un diseño factorial mixto 2 x 2 siendo el primer factor de grupos independientes, grupo de intervención frente a grupo control y un segundo factor de medidas repetidas con dos niveles (evaluación pre-intervención frente evaluación post- intervención). Se trata por tanto de un diseño cuasiexperimental de comparación entre grupos con medición pretest- postest con un grupo control y un grupo experimental.

Las variables dependientes fueron, las puntuaciones referidas a los instrumentos, ASI (Índice de sensibilidad a la ansiedad), MAAS (escala de atención consciente) y la subescala de ansiedad del SCL-90-90-R (grado de malestar psicológico).

La variable independiente es el programa de reducción del estrés basado en mindfulness (MBSR).

\section{Procedimiento}

La captación de los participantes se llevó a cabo mediante la publicación de carteles en diferentes centros de salud y farmacias de la provincia de Almería. Se les invitaba a participar voluntariamente en los talleres con el fin de reducir el estrés y la ansiedad. Una vez que contactaron, se les reunió a todos para explicarles la finalidad y metodología de los talleres y el anonimato de las puntuaciones en los cuestionarios.Siendo todos ellos mayores de edad y queriendo participar voluntariamente en el estudio, se les administró una serie de cuestionarios (ASI-3, MAAS y Subescala de Ansiedad del SCL-90). Se hizo una criba con aquellos participantes que puntuaron alto en los cuestionarios (ASI-3 y SCL-90). De tal manera, que los participantes que formaron parte del grupo control o del grupo experimental fueron aquellos que obtuvieron puntuaciones elevadas en los cuestionarios de ASI-3 y SCL-90. De un total de 35 asistentes, finalmente quedaron 20 sujetos (aquellos que puntuaron alto en el ASI-3 y SCL90), las pruebas fueron administradas por las investigadoras, licenciadas en psicología. Concluyendo que de los 35 asistentes aquejados de ansiedad y que se interesaron por nuestra oferta de intervención, 20 de ellos tenían ansiedad en ese momento. 
Los participantes del grupo experimental asistieron a unos talleres basados en la metodología del programa de reducción del estrés basado en mindfulness (MBSR) (KabatZinn, 2003), durante ocho sesiones semanales de dos horas de duración (ver Tabla 1). Desde la primera sesión se instauró un plan diario de mindfulness. Además se promovió una intención de generalización de las claves propias de la meditación mindfulness a la vida cotidiana de los participantes, vivenciación de los síntomas de ansiedad en sesión con el fin de aceptarlos y quitarles protagonismo en la vida de la persona. Cada sesión de entrenamiento constaba en la práctica de diferentes ejercicios basados en focalizar la atención sobre la respiración y sobre las sensaciones corporales, relajación y meditación con el fin de aprender a relacionarse con los eventos privados dejándolos fluir, de tal manera, que se promoviese la aceptación, abandonando el control y la lucha contra aquellos eventos internos molestos. Siendo este procedimiento una reestructuración cognitiva desde la perspectiva demindfulness. Los participantes del grupo control, asistieron a talleres sobre diversas técnicas de relajación como relajación guiada o relajación en imaginación y a través de la música, en las que se practicaban diversos estiramientos musculares, también se hablaba sobre la ansiedad y las diferentes experiencias de los participantes. Este grupo trabajó durante 8 sesiones de dos horas de duración cada una. Fue por tanto un grupo control activo.

Ambos grupos (experimental y control) estuvieron guiados por dos psicólogas. La intervención se realizó, los viernes por la tarde (para el grupo experimental) y los sábados por la mañana (para el grupo control) en una sala diáfana, amplia y ventilada.

Análisis de los datos.

Para analizar la existencia de diferencias estadísticamente significativas en las medidas pretest y postest entre ambos grupos, se utilizó la prueba $t$ de Student para muestras independientes. En el segundo análisis se compararon las medidas postest con las pretest, de cada grupo, utilizando la $t$ de Student para muestras relacionadas. En tercer lugar, se utilizó la $d$ de Cohen (1988) para valorar la magnitud del cambio producida por la intervención y finalmente, se calculó el porcentaje de cambio entre las puntuaciones postest y pretest de ambos grupos. Para la realización de todos los análisis estadísticos se empleó el paquete estadístico SPSS, versión 19.0.

\section{Resultados}

En primer lugar, se hallaron las puntuaciones medias y las desviaciones típicas para cada una de las variables del estudio; sensibilidad a la ansiedad (ASI-3), mindfulness (MAAS), y subescala de ansiedad del (SCL-90) correspondientes a los grupos control y experimental en la fase pretest y postest (véase la Tabla 2).

En el primer análisis, tan solo parecieron diferencias significativas en las medidas pretest entre ambos grupos (experimental y control) en la variable ansiedad social, (Tabla 3). En las medidas postest, se encontraron diferencias estadísticamente significativas en la puntuación total de sensibilidad a la ansiedad y en todos sus factores (sensibilidad a la ansiedad física, cognitiva y social). En la variable atención consciente se encontraron diferencias 
significativas, entre el grupo control y experimental. En la subescala de ansiedad del SCL-90 se hallaron diferencias estadísticamente significativas entre ambos grupos.

Tabla 2. Medias (M) y desviaciones típicas (DT) pretest y postest correspondientes a los grupos control y experimental, para las variables sensibilidad a la ansiedad, escala atención consciente (MAAS) y síntomas de ansiedad (SCL-90).

\begin{tabular}{|c|c|c|c|c|c|c|c|c|}
\hline & \multicolumn{4}{|c|}{$P R E$} & \multicolumn{4}{|c|}{ POST } \\
\hline & \multicolumn{8}{|c|}{ EXPERIMENTAL CONTROL EXPERIMENTAL CONTROL } \\
\hline & M & DT & M & DT & M & DT & M & DT \\
\hline Asi-fis & 10,40 & 2,17 & 9,7 & 2,58 & 3,90 & 1,79 & 8 & 2,7 \\
\hline Asi-cog & 10,3 & 4,19 & 10,2 & 4,18 & 3,8 & 2,14 & 8,1 & 4,09 \\
\hline Asi-soc. & 12,8 & 1,81 & 15,5 & 3,37 & 3,6 & 2,31 & 14,2 & 3,45 \\
\hline Asi-total & 33,5 & 6,94 & 35,4 & 4,32 & 11,3 & 5,61 & 30,3 & 5,29 \\
\hline Maas & 37,3 & 6,6 & 33,3 & 4,84 & 57,8 & 8,18 & 33,9 & 7,18 \\
\hline Scl-90 & 31,9 & 5,4 & 30,2 & 3,01 & 18,00 & 2,86 & 28,4 & 2,27 \\
\hline
\end{tabular}

En el segundo análisis, el grupo control no presentó diferencias significativas en ninguna de las variables ni en sus factores, mientras que en el experimental aparecieron diferencias estadísticamente significativas, tanto en las puntuaciones globales del ASI-3 como en todos sus factores, ansiedad física, ansiedad cognitiva y ansiedad social. Ocurriendo de manera parecida en el inventario MAAS y en la subescala de ansiedad del SCL-90 (véase Tabla 4).

Tabla 3. Prueba t de Student para muestras independientes de las diferencias pretest y postest entre el grupo control y experimental, para las variables sensibilidad a la ansiedad, atención consciente y síntomas de ansiedad.

\begin{tabular}{lcccc}
\hline & \multicolumn{2}{c}{ PRE } & \multicolumn{2}{c}{ POST } \\
\hline Variable & $t$ & $p$ & $t$ & $p$ \\
\hline Asi-fis & 0,262 & 0,791 & $-3,73$ & $0,002^{*}$ \\
Asi-cog & 0,329 & 0,746 & $-2,95$ & $0,008^{*}$ \\
Asi-social & $-2,77$ & $0,013^{*}$ & $-10,54$ & $0,000^{* *}$
\end{tabular}




\begin{tabular}{lcccc} 
Asi-total & $-1,05$ & 0,308 & $-7,48$ & $0,000 * *$ \\
Maas & 1,84 & 0,081 & 7,65 & $0,000 * *$ \\
Scl-90 & 2,51 & 0,221 & $-11,02$ & $0,000 * *$ \\
\hline Nota; & ${ }^{*} p<0,05$, & ${ }^{* *} p<0,01 ;$ & $* * * p<0,001$ &
\end{tabular}

Para valorar la magnitud del cambio una vez finalizada la intervención, se empleó la $d$ de Cohen (1988). Valores superiores a.8 indican cambios muy importantes, entre 0,5 y 0,8 importantes, por debajo de 0,5 medios y menores de 0,2 bajos. Las puntuaciones de la $d$ de Cohen (Tabla 5) en el grupo control muestran la existencia de cambios pequeños, con un rango entre 0,04 y 0,03 mientras que el grupo experimental presentó puntuaciones en un rango entre 0,69 y 0,86 , lo que indica que se produjeron cambios importantes.

Tabla 4. Prueba t de Student para muestras relacionadas de las diferencias postest-pretest en el grupo control y experimental, para las variables sensibilidad a la ansiedad, atención consciente y síntomas de ansiedad.

\begin{tabular}{llccc}
\hline & \multicolumn{2}{c}{ CONTROL } & \multicolumn{2}{c}{ EXPERIMENTAL } \\
\hline Variable & $t$ & $p$ & $t$ & $p$ \\
\hline Asi-fis & $-2,31$ & $0,05^{*}$ & $-6,28$ & $0,000^{* * *}$ \\
Asi-cog & $-2,2$ & 0,06 & $-4,21$ & $0,000^{* * *}$ \\
Asi-soc. & $-2,46$ & $0,04^{*}$ & $-8,84$ & $0,000^{* * *}$ \\
Asi-total & $-3,59$ & $0,01^{* *}$ & $-6,96$ & $0,000^{* * *}$ \\
Maas & $-0,16$ & 0,88 & $-5,16$ & $0,000^{* * *}$ \\
Scl-90 & $-0,21$ & $0,000^{* * *}$ & $-8,72$ & $0,000^{* * *}$
\end{tabular}

Nota; ${ }^{*} p<0,05 ;{ }^{* *} p<0,01 ; * * * p<0,001$

En el grupo experimental, en la variable sensibilidad a la ansiedad se produce un cambio grande o muy importante en la puntuación global de Sensibilidad a la ansiedad $(d=0,86)$ y en sus subescalas encontramos cambios importantes en el factor Sensibilidad a la ansiedad física (temor de sentir síntomas físicos de ansiedad, con una $(d=0,85)$, cambios moderados en Sensibilidad a la ansiedad cognitiva (temor a síntomas cognitivos de ansiedad, $(d=0,69) \mathrm{y}$ cambios muy importantes en Sensibilidad a la ansiedad social (temor a experimentar síntomas de ansiedad en situaciones sociales) $(d=0,91)$. En la escala MAAS se produjeron cambios grandes o muy importantes $(d=0,8)$ y también en la subescala de ansiedad del Scl-90 (experimentación de síntomas de ansiedad, con una $d=0,84$ ). 
Tabla 5. D de Cohen y porcentaje de cambio de las variables de sensibilidad a la ansiedad, atención consciente y síntomas de ansiedad.

\begin{tabular}{lcccc}
\hline \multicolumn{3}{c}{ Magnitud del cambio } & \multicolumn{3}{c}{ \% de cambio } \\
\hline & EXPERIMENTAL & CONTROL & EXPERIMENTAL & CONTROL \\
\hline Asi-fis & 0,85 & 0,32 & 47 & 21,3 \\
Asi-cog & 0,69 & 0,24 & 43 & 14,7 \\
Asi-social & 0,91 & 0,18 & 51,6 & 7,7 \\
Asi-total & 0,86 & 0,46 & 47,4 & 27,4 \\
Maas & 0,82 & 0,04 & 47,6 & 0 \\
Scl-90 & 0,75 & 0,34 & 38,3 & 23,9
\end{tabular}

En el grupo control se observan cambios poco importantes, en la puntuación global de Sensibilidad a la ansiedad $(d=0,46)$ y en sus subescalas, en el factor Sensibilidad a la ansiedad física $(d=0,3)$, en Sensibilidad a la ansiedad cognitiva $(d=0,24)$ y en Sensibilidad a la ansiedad social $(d=0,18)$. En la escala MAAS no se produjeron cambios $(d=0,04)$ y cambios pequeños o poco importantes también en la subescala de ansiedad de SCL-90 $(d=0,3)$.

\section{Discusión}

Con los resultados del presente estudio se confirma la hipótesis que nos planteábamos en la presente investigación. Los sujetos del grupo experimental obtuvieron una reducción significativa de las medidas de sensibilidad a la ansiedad (ASI-3) y síntomas de ansiedad (SCL90), y una mejora en MAAS en comparación con los sujetos del grupo control. Ambos grupos partieron de puntuaciones similares en dichas variables, ya que se establecieron los grupos de manera aleatoria y no se obtuvieron diferencias significativas entre las puntuaciones pretest de ambos grupos, pero una vez finalizada la intervención basada en mindfulness en el grupo experimental se observaron diferencias significativas entre las puntuaciones postest de los dos grupos en dos de los factores generales. Además, la variable independiente, produjo una disminución en las puntuaciones de sensibilidad a la ansiedad y ansiedad en el grupo experimental al comparar sus puntuaciones pre y post, aspecto que no ocurrió al analizar los datos pretest-postest del grupo control. Son destacables la magnitud de los cambios y los porcentajes de cambio en el grupo experimental, como consecuencia de los efectos del programa de entrenamiento, en comparación con el grupo control.

Estos datos concuerdan con los hallazgos de autores como Evans et al. (2008) y Roemer y Orsillo (2007), quienes constatan mejoras significativas en los niveles de ansiedad en grupos 
no controlados con personas con ansiedad generalizada mediante un programa de tratamiento basado en mindfulness. Por otro lado,Rasmussen y Pidgeon (2011) encuentran resultados positivos en el trastorno de ansiedad social.

A su vez los resultados de este estudio concuerdan con los encontrados en otras investigaciones en estudios controlados aleatorizados, donde Roemer et al. (2008) encontraron mejoras en los niveles de ansiedad, preocupación, evitación de las emociones, calidad de vida y en mindfulness.Del mismo modo, otras investigaciones sugieren que mindfulness está relacionado con multitud de aspectos relacionados con la mejora de la salud psicológica, específicamente, ciertos aspectos inherentes a mindfulness como la autocompasión, la flexibilidad cognitiva, la aceptación y el no enjuiciamiento de los eventos privados(Woodruff, Glass et al. 2014).

Sin embargo, Goyal et al. (2014), a través de un meta-análisis ofrecen resultados distintos en cuanto a la efectividad de mindfulness, ya que encuentran unas mejoras moderadas en los niveles de ansiedad, depresión y dolor cuando se compara con grupos controles activos no específicos. Abogando por la necesidad de un mayor número de investigaciones sobre mindfulness con diseños experimentales más sólidos que permitan arrojar conclusiones fiables en cuanto a la eficacia de mindfulness y las situaciones y casuísticas que facilitan unos resultados óptimos de los programas basados en mindfulness.

Los datos del presente estudio, aunque positivos, hay que analizarlos con la debida cautela. Entre sus limitaciones está el que la muestra de la investigación fue reducida, por lo que se pueden presentar sesgos. No se controló si los sujetos de uno u otro grupo hubieran estado en ese momento tomando psicofármacos, lo cual podría haber influido notablemente en los resultados. Además, no se ha realizado un seguimiento, por lo que será necesario confirmar en investigaciones posteriores si las mejoras obtenidas en el grupo experimental, mediante la participación en el programa de mindfulness, se mantienen a lo largo del tiempo mediante la realización de diversas medidas de seguimiento. Siendo necesaria la realización de más estudios con una muestra más amplia para corroborar lo que parece apuntar esta investigación. Tal y como afirman Goyal et al. (2014), es preciso observar los resultados de los programas basados en mindfulness después de un año, ya que mindfulnesses una habilidad que se aprende e interioriza con la práctica, esperando que sea tras un periodo de tiempo cuando aparezcan realmente los verdaderos beneficios. Tal y como señalan otros autores, el cultivo de mindfulness correlaciona con el incremento de la salud psicológica (Nyklicek y Kuijpers, 2008).

\section{Referencias}

Baer, R. (2003). Mindfulness training as a clinical intervention: A conceptual and empirical review. Clinical Psychology: Science and Practice, 10, 125-142.

Baer, R. A. (Ed.). (2006). Mindfulness-based treatment approaches: Clinicians guide to evidence base and practice. New York: Academic Press.

Barnes, V. A., Treiber, F. A. y Davis, H. (2001). Impact of transcendental meditation on cardiovascular function at rest and during acute stress in adolescents with high normal blood pressure.Journal of Psychosomatic Research, 51, 597-605. 
Brown, K. W. y Ryan, R. M. (2003). The benefits of being present: The role of mindfulness in psychological well-being. Journal of Personality and Social Psychology, 84, 822-848.

Chiesa, A. y Serretti, A. (2009). Mindfulness-based stress reduction for stress management in healthy people: A review and meta-analysis. The Journal of Alternative and Complementary Medicine, 15, 593-600.

Evans, S., Fernando, S., Findler, M. Stowell, C., Smart, C., y Haglin, D. (2008). Mindfulnessbased cognitive therapy for generalized anxiety disorder. Journal of anxiety disorders, 22, 716-721.

Franco, C., Mañas, I., Cangas, A., Moreno, E. y Gallego, J. (2010). Reducing teachers' psychological distress through a mindfulness training program. The Spanish Journal ofPsychology, 13, 2,655-666.

Franco, C., Mañas, I., y Justo, E. (2010). Mejora en algunas dimensiones de salud percibida en pacientes con fibromialgia mediante la aplicación de un programa de meditación mindfulness. Psychology, Society and Education, 2, 2, 117-130.

Germer, C. K., Siegel, R. D. y Fulton, P. R. (2005). Mindfulness and psychotherapy. Nueva York: Guilford Press.

Goyal, M., Singh, S., Sibinga, E. M. S., Gould, N. F., Rowland-Seymour, A., Sharma, R., . . .Haythornthwaite, J. A.(2014). Meditation programs for psychological stress and wellbeing: A systematic review and meta-analysis. Journal of American Medical Association Internal Medicine, 174, 357-368.

Grossman, P., Niemann, L., Schmidt, S. y Walach, H. (2004). Mindfulness-based stress reduction and health benefits: A meta-analysis. Journal of Psychosomatic. Research, 57, 35-43.

Hayes, S. C., Luoma, J. B., Bond, F. W., Masuda, A., y Lillis, J. (2006). Acceptance and Commitment Therapy: Model, Processes and Outcomes. Behaviour Research and Therapy, 44, 1-25.

Hayes, S. C. (2004a). Acceptance and commitment therapy and the new behaviour therapies.

En S. C. Hayes, V. M. Follette y M. M. Linehan (Eds.), Mindfulness and acceptance:

Expanding the cognitive behavioral tradition (pp. 1-29). Nueva York: The Guilford Press.

Hayes, S. C. (2004b). Acceptance and commitment therapy, relational frame theory, and the

third wave of behavioral and cognitive therapies. Behavior therapy, 35, 639-665.

Irving, J. A., Dobkin, P. y Park, J. (2009). Cultivating mindfulness in health care professionals: A review of empirical studies of Mindfulness-Based Stress Reduction (MBSR) program.Complementary Therapies in Clinical Practice, 15, 61-66.

Jacobs, G. D., Friedman, R. y Benson, H. (1993). Home- based central nervous system assessment of a multifactor behavioral intervention for chronic sleep-onset insomnia. Behavior therapy, 24, 159-174.

Kabat-Zinn, J. (1990). Full catastrophe living. Nueva York: Delacorte.

Kabat-Zinn, J. (1994). Wherever you go, there you are: Mindfulness meditation in everyday life. New York: Hyperion.

Kabat-Zinn, J. (2003). Vivir con plenitud las crisis. Cómo utilizar la sabiduría del cuerpo y la mente para afrontar el estrés, el dolor y la enfermedad. Barcelona: Kairós.

Kabat-Zinn, J. (2005). Coming to Our Senses. New York, NY: Hyperion. 
Kim, Y. W., Lee, S. H., Choi, T. K., Suh, S. Y., Kim, B., Kim, C. M., Cho, S. J., Kim, M. J., Yook, K., Ryu, M., Song, S. K. y Yook, K. H. (2009). Effectiveness of mindfulnessbased cognitive therapy as an adjuvant to pharmacotherapy in patients with panic disorder or generalized anxiety disorder. Depression and Anxiety, 26, 7,601-606.

Lykins, E. L. y Baer, R. A. (2009). Psychological functioning in a simple of long- term practitioners of mindfulness mediation. Journal of cognitive Psychotherapy, 23, 226241.

Mañas, I. (2007). Nuevas terapias psicológicas: La tercera ola de terapias de conducta o terapias de tercera generación. Gaceta de Psicología, 40, 26-34.

Mañas, I., Sánchez, L. C. y Luciano, M. C. (2008). Efectos producidos por un ejercicio de mindfulness (body-scan): Un estudio piloto. Póster presentado en el VII Congreso de la Sociedad Española de Psicología Experimental, San Sebastián-Donostia, España.

Mañas, I., Luciano, M. C. y Sánchez, L. C. (2008). Beginners practising a basic mindfulness technique: An experimental analysis. Comunicación presentada en la $4{ }^{\text {th }}$ Conference of the European Association for Behaviour Analysis, Madrid.

Mañas, I., Franco, C. y Justo, E. (2011). Reducción de los niveles de estrés docente y los días de baja laboral por enfermedad en profesores de enseñanza secundaria obligatoria. A través de un programa de entrenamiento en mindfulness. Clínica y salud, 22, 2, 121-137.

Marks, I. (1986). Tratamiento de neurosis. Barcelona: Martínez Roca.

Miró, M.,T., Perestelo, L., Pérez, J., Giménez, S., Rivero, A., González, M. (2009).Eficacia de los tratamientos psicológicos basados en la Atención Plena en el tratamiento de la depresión y la ansiedad. Plan Nacional para el Informe de Evaluación de Tecnologías Sanitarias: SESCS No 2007/11. Servicio de Evaluación del Servicio Canario de la Salud. Sistema Nacional de Salud del Ministerio de Sanidad y Política Social.

.Nyklicek, I. y Kuijpers, K., J. (2008). Effects of mindfulness- based stress reduction intervention on psychological well-being and quality of life: Is Increased Mindfulness Indeed the Mechanism?.Annals of Behavioral Medicine. 35, 3, 331-340.

Orsillo, S. M., Roemer, L., Block-Lerner, J., LeJeune, C. y Herbert, J. D. (2004). ACT with anxiety disorders. En S. C. Hayes y K. Strosahl (Eds.), A practical guide to acceptance and commitmen therapy (pp. 103-132). Nueva York: Springer.

Orsillo, SM, Roemer L, Lerner JB, Tull MT. (2004). Mindfulness and acceptance: expanding the cognitive-behavioral tradition. En S.C. Hayes, V. M. Follette, M. M. Linehan, (Eds.), Acceptance, mindfulness, and cognitive-behavioral therapy: comparisons, contrasts, and application to anxiety (pp 66-95). Nueva York: Guilford.

Pérez-Álvarez, M. (2008). Las terapias de conducta de tercera generación como modelo contextual de psicoterapia. FOCAD Formación Continuada a Distancia, 2, 10-16.

Prieto, J. M. (2007). Psicología de la meditación, la psique de vuelta a casa. InfocopOnline Revista de Psicología, 33.

Rasmussen, M., K. y Pidgeon, A.,M. (2011). The direct and indirect benefits of dispositional mindfulness on sel-esteem and social anxiety. Anxiety Stress Coping, 24, 2, 227-233.

Reiss, S. y McNally, R. J. (1985). Expectancy model of fear.En S. Reiss y R. Bootzin(Eds.), Theoretical issues in behavior therapy (pp. 107-121). San Diego: Academic Press. 
Reiss, S., Peterson, R. A., Gursky, M. y McNally, R. J. (1986). Anxiety, sensitivity, anxiety frequency, and the prediction of fearfulness.Behaviour Research and Therapy, 24, 1-8.

Reiss, S. (1991). Expectancy model of fear, anxiety and panic. Clinical Psychology Review, 11, 141-153.

Roemer, L. y Orsillo, S. M. (2004). Acceptance-based behavior therapy for GAD: Preliminary findings from an open trial and a randomized controlled trial.Acceptance-based behavioral therapies: New directions in treatment development across the diagnostic spectrum. Symposium presented at the 38 the annual meeting of the Association for Advancement of Behavior Therapy, New Orleans, LA.

Roemer L. y Orsillo S. M., (2003).Mindfulness: A Promising Intervention strategy in need of further study. Clinical psychology: Science and practice, 10, 172-178.

Roemer, L. y Orsillo, S. M. (2007). An open trial of an acceptance-based behavior therapy for generalized anxiety disorder. BehaviorTherapy, 38,72-85.

Sandín, B., Valiente, R.M. y Chorot, P. (1999). Material de apoyo para la evaluación de los miedos y fobias. En B. Sandín (Ed.), Las fobias específicas (pp. 161-188). Madrid: Klinik.

Sandín, B., Valiente, R. M., Chorot, P. y Santed, M. A. (2005). Propiedades psicométricas del índice de sensibilidad a la ansiedad. Psicothema, 17, 3, 478-483.

Solberg, E. E., Ingjer, F., Holen, A., Sundgot-Borgen, J., Nilsson, S. y Holme, I. (2000). Stress reactivity to and recovery from a standardised exercise bout: A study of 31 runners practising relaxation techniques. British Journal of Sports Medicine, 34, 268-272.

Thyer, B. A. (1987). Treating anxiety disorders: A guide for human service professionals.London: Sage Publications.

Vallejo, M. A. (2007). El mindfulness y la "tercera generación de terapias psicológicas”. Infocop, 33, 16-18.

Vila, J. (1984). Técnicas de reducción de ansiedad. In J. Mayor \& F. J. Labrador (Eds.), Manual de Modificación de conducta (pp. 229-264). Madrid: Alhambra.

Williams, J. M. G. (2010). Commentary.Mindfulness and psychological process. Emotion, 10, $1,1-7$. 
ANEXO.

Tabla 1. Esquema general de la intervención.

Sesión Ejercicios

1 Presentación de los miembros del grupo y exposición de los motivos para participar en el curso y las expectativas que poseen.

Exposición del concepto de mindfulnessy condiciones previas necesarias para comenzar a meditar.

Explicación de la práctica de meditación mindfulness.

Realización de la $1^{\text {a }}$ práctica de meditación.

2 Ejercicio de tratar de no pestañear durante un minuto y analogías relacionadas con el control de eventos privados.

Ejercicio de exploración corporal (bodyscan).

Diez minutos de meditación sentado prestando atención plena a la respiración.

3 Entrenamiento en el reconocimiento de nuestras propias emociones y de la conexión existente entre nuestro cuerpo y nuestra mente.

Yoga consciente de pie, prestar atención plena a la respiración y prestar atención plena a la respiración y al cuerpo.

Ejercicio de atención a las sensaciones de la cara.

$4 \quad$ Concepto de reaccionar en lugar de responder a las situaciones.

Reflexión sobre los siguientes conceptos: automatismo, estancamiento, percepción, emoción, reacción y afrontamiento.

Yoga consciente de pie, observación de sensaciones en la zona del pecho y abdomen.

Tomar consciencia de sentimientos agradables/desagradables.

$5 \quad$ Explicación de los conceptos de patrón de respuesta, reacción y sus efectos en la salud.

Estrategias alternativas de afrontamiento del dolor basadas en mindfulness.

Realización de prácticas de yoga y meditación mindfulness.

Prestar atención plena a la respiración y prestar atención plena a la respiración y al cuerpo

y, a continuación, explorar una dificultad.

Respiración de 3 minutos observando las sensaciones de los brazos

6 Entrenamiento en habilidades de comunicación. Expresar pensamientos y sentimientos de manera saludable.

Realización de prácticas de yoga y meditación mindfulness.

Prestar atención plena a la respiración, al cuerpo, a los sonidos y los pensamientos.

Respiración de tres minutos observando las sensaciones de las piernas.

$7 \quad$ Concepto de alimentación saludable e incorporación a nuestra vida diaria.

Realización de prácticas de yoga y meditación mindfulness.

Ejercicio de exploración corporal.

8 Entrenamiento y generalización de las técnicas aprendidas en situaciones de malestar.

Responsabilidad en el auto-cuidado.

Elegir una pauta de práctica formal e informal de atención plena que pueda mantener el resto de su vida. 